\title{
Exploring metabolomic changes due to cortisol deficiency in early development using the ferredoxin ( $\mathrm{fdx} 1 \mathrm{~b}$ ) null-allele zebrafish
}

\author{
Meltem Weger ${ }^{1}$, Aliesha Griffin ${ }^{1}$, Benjamin Gorling ${ }^{2}$, Angela E. Taylor ${ }^{3}$, Burkhard Luy², Ferenc Mueller ${ }^{1}$, Nils Krone ${ }^{3,4}$ \\ ${ }^{1}$ Institute of Cancer and Genomic Sciences, ${ }^{3}$ Institute of Metabolism and Systems Research, University of Birmingham; ${ }^{2}$ Institute for Organic Chemistry, Karlsruhe Institute of \\ Technology; ${ }^{4}$ Department of Human Metabolism, University of Sheffield
}

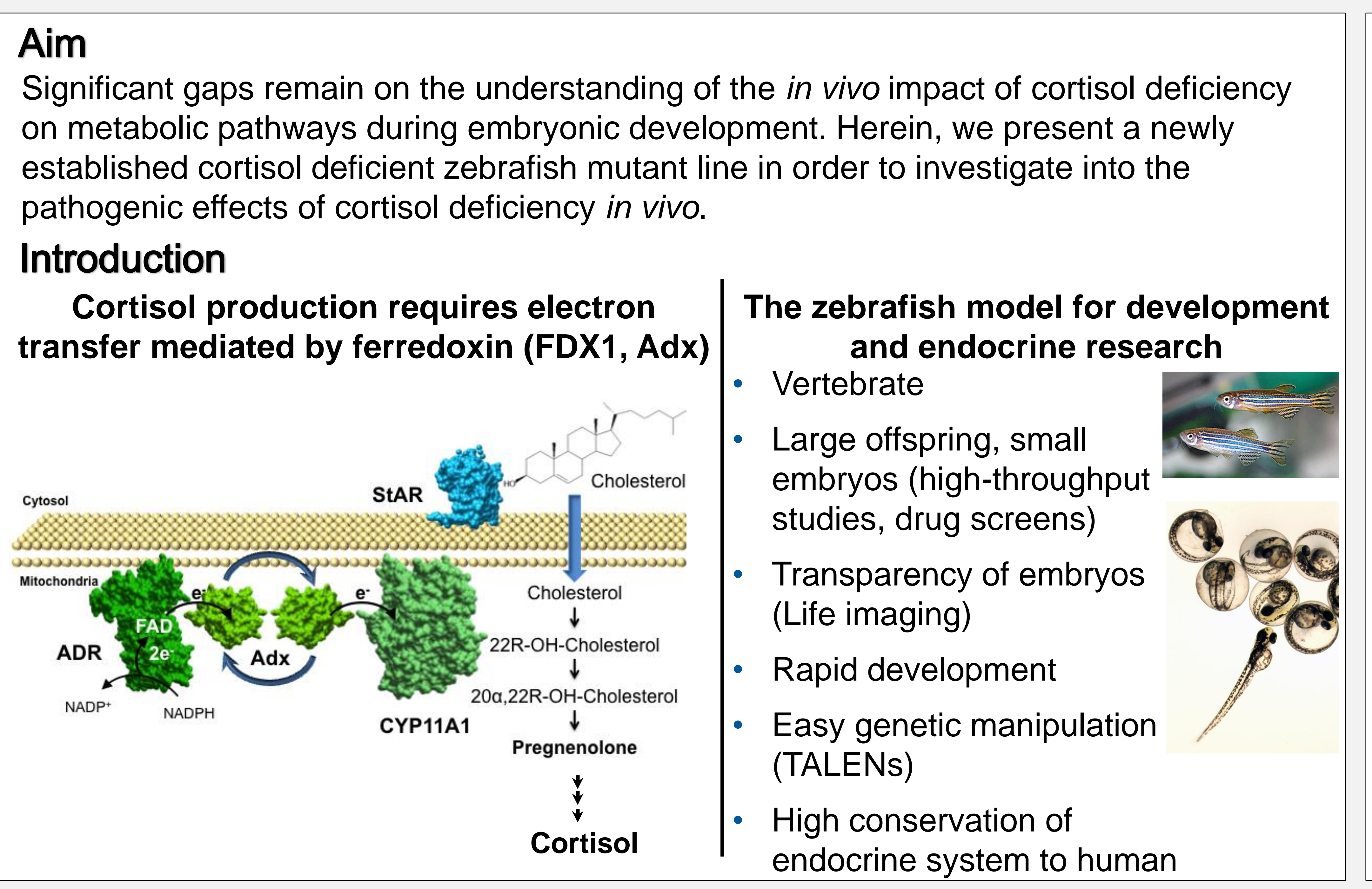

\section{Summary}

- We have generated a mutant $f d x 1 b$ (equivalent of human FDX1) null-allele zebrafish line

- $f d \times 1 b$ deficient embryos are darker due to a failure in Visual Background Adaptation (VBA) behaviour

- VBA in the fdx1b mutants is rescued after dexamethasone treatment

pomc expression is significantly increased in $\mathrm{fd} \times 1 \mathrm{~b}$ null-allele larvae

- Cortisol synthesis and signalling are significantly impaired

- $f d x 1 b$ null-allele larvae have a blunted cortisol response to stress

- Metabolic profiling reveals changes in energy synthesis and biomolecule generation

\section{Conclusion}

The $\mathrm{fd} \times 1 \mathrm{~b}$ null-allele zebrafish line is a promising in vivo model to explore the pathophysiologic impact of glucocorticoid deficiency on energy metabolism relevant to early development and potentially adult life.

\section{Results}

fdx1b null-allele zebrafish larvae reveal a failure in their Visual Background Adaptation (VBA) behaviour
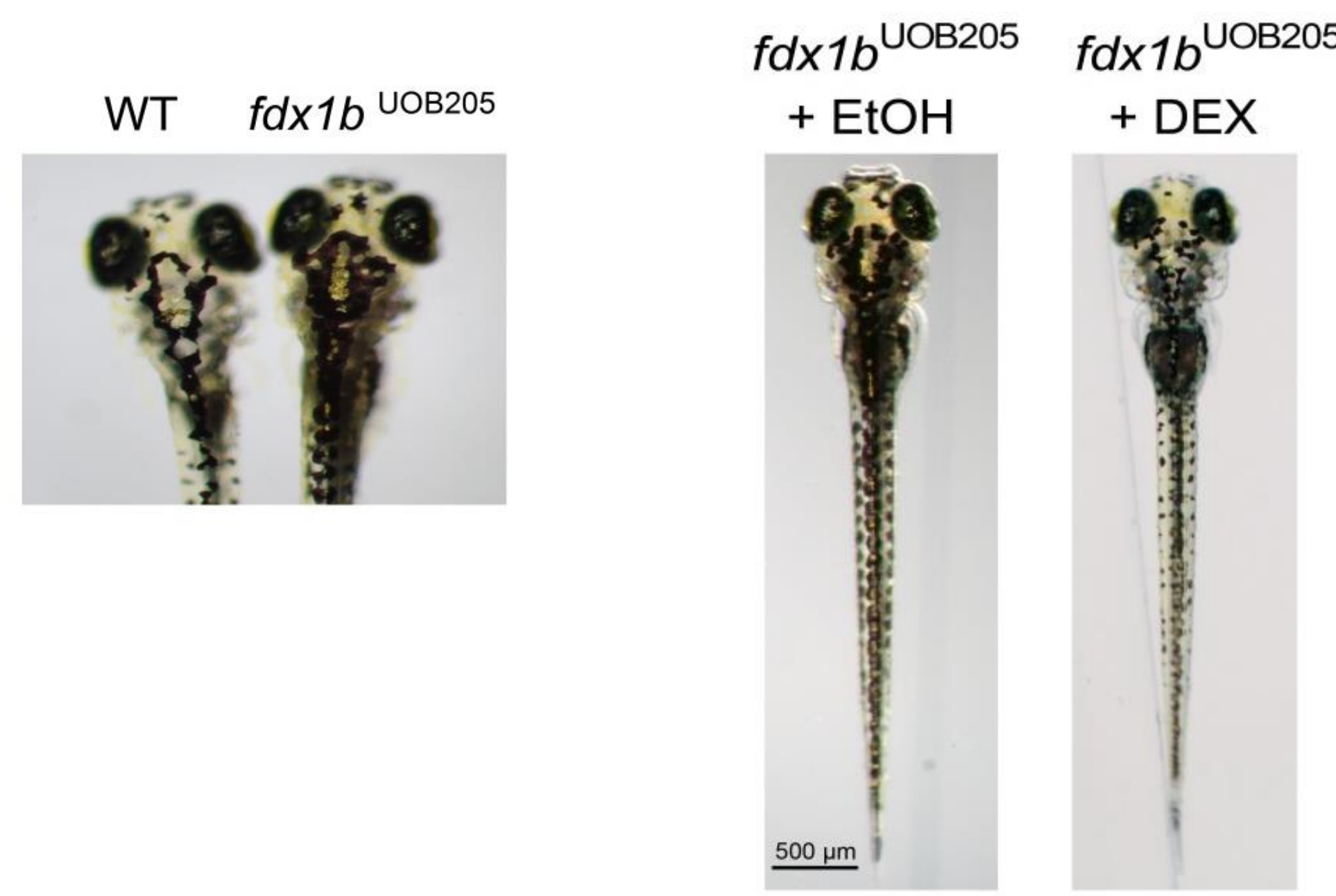

fdx1b null-allele zebrafish larvae are impaired in cortisol synthesis, cortisol regulated gene expression and in their stress response
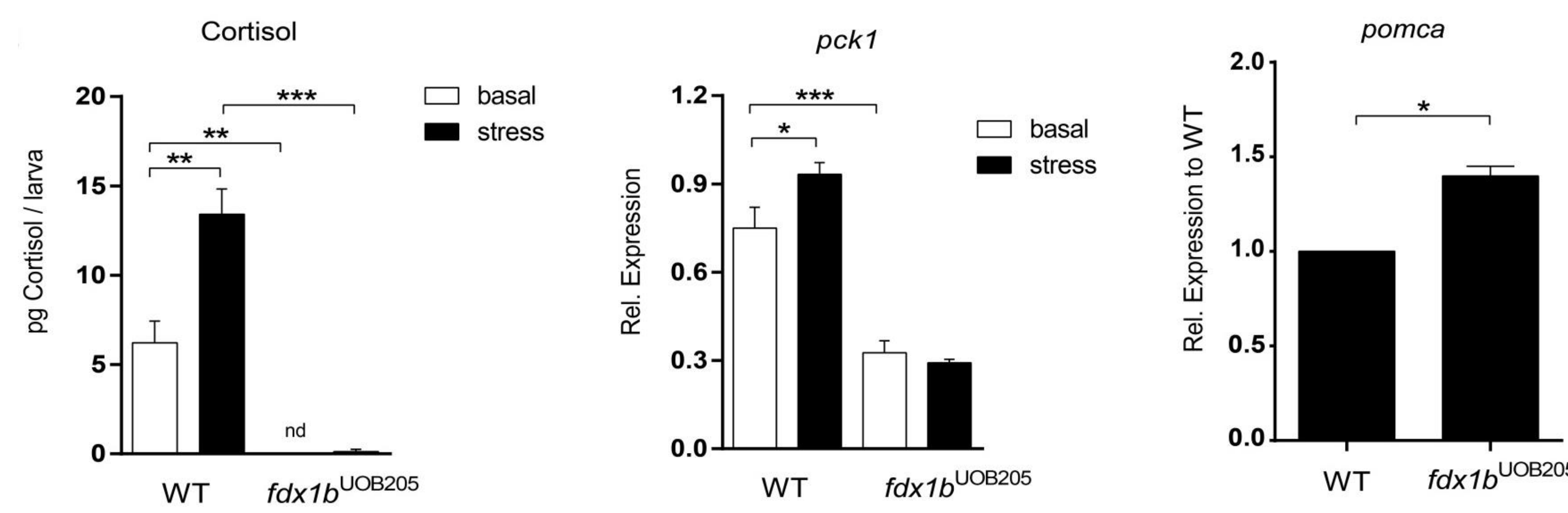

\section{Cortisol deficiency leads to metabolic changes in pathways involved in} energy and biomolecule synthesis

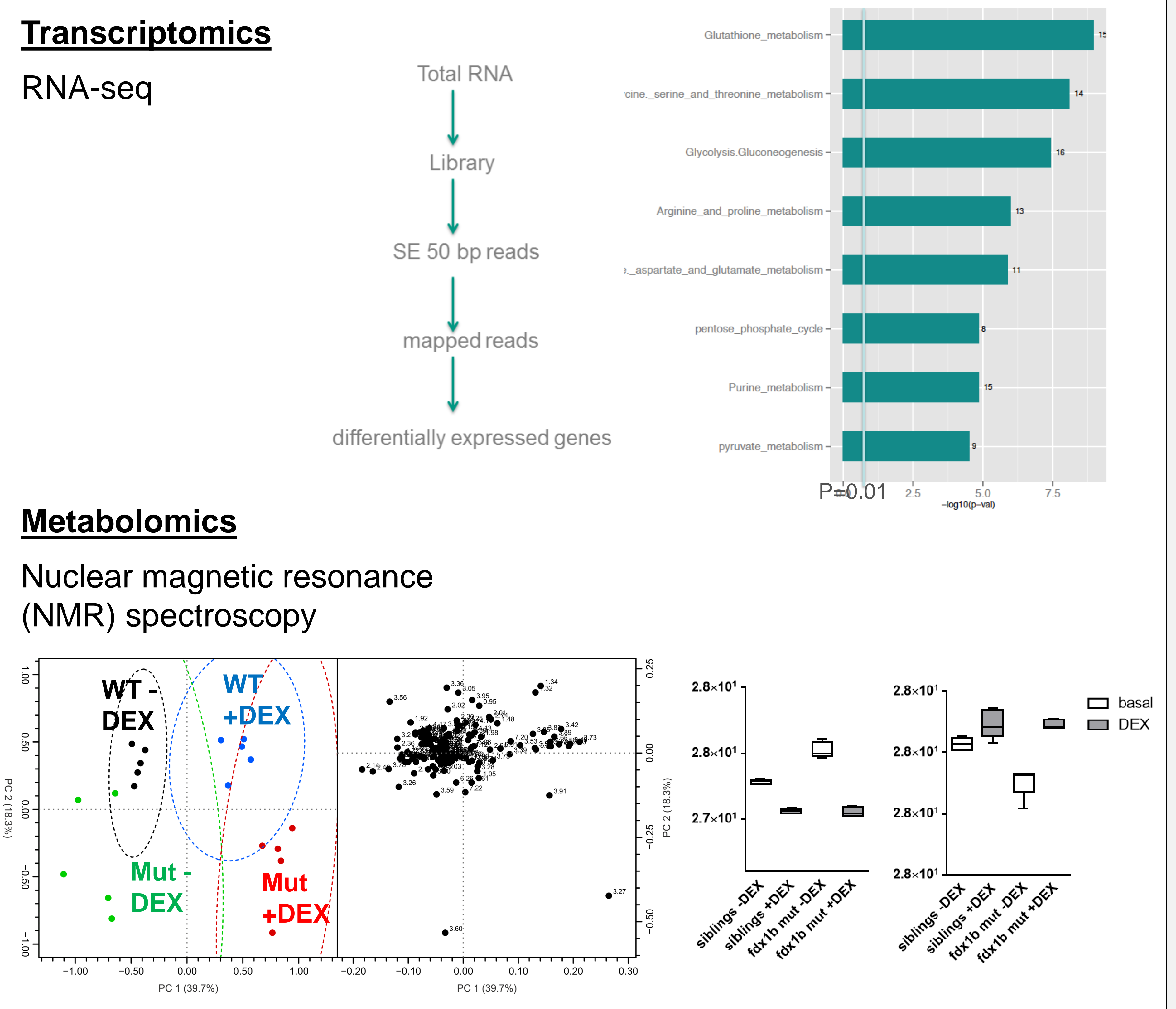

\section{Material and methods}

Establishing a fdx1 null-allele zebrafish mutant line using Transcription Activator-like Effector Nucleases (TALENs)

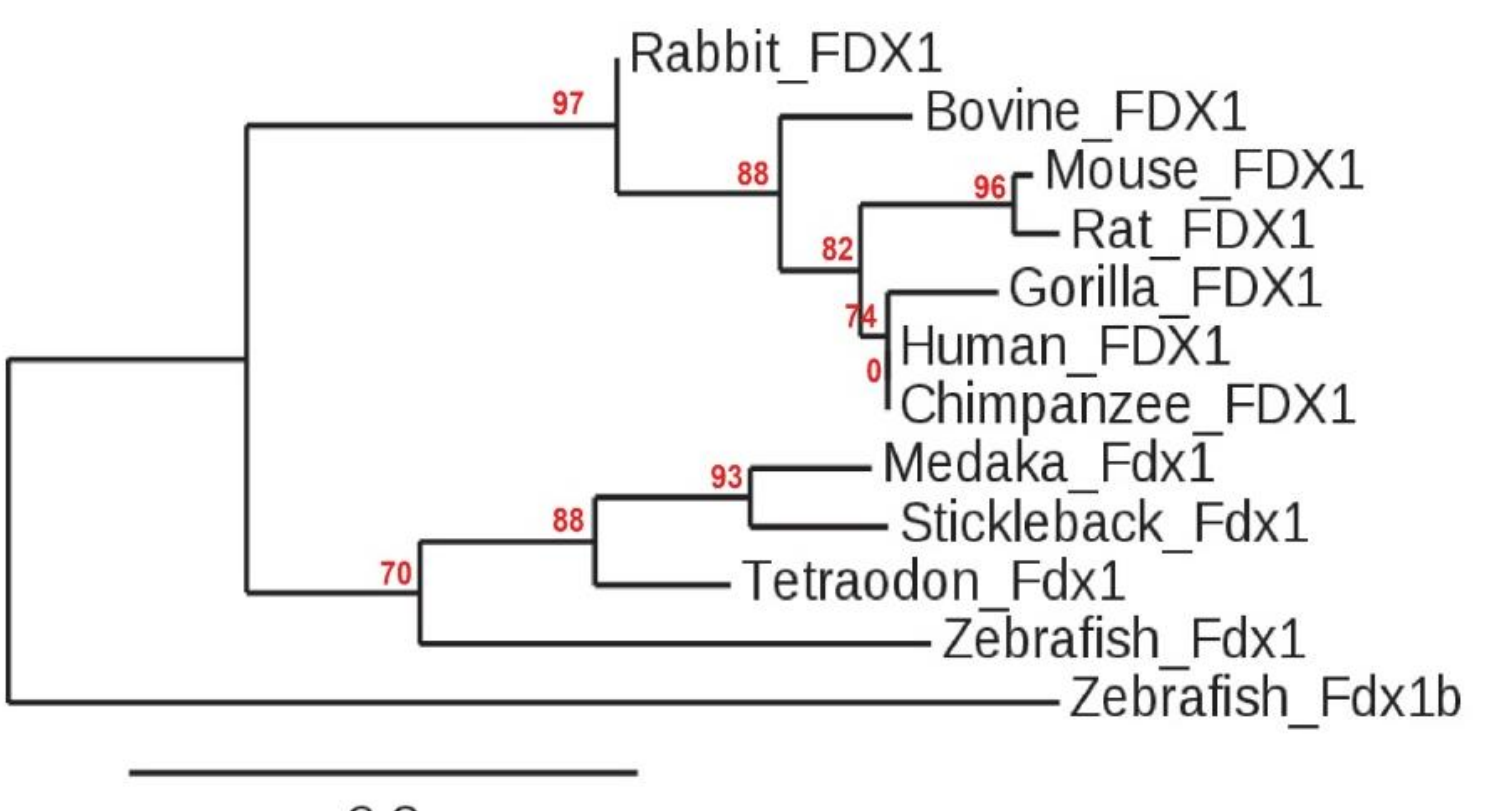

From the duplicated zebrafish $\mathrm{fd} \times 1$ genes (fdx 1 , $\mathrm{fd} \times 1 \mathrm{~b}), \mathrm{fdx} 1 \mathrm{~b}$ is mediating cortisol synthesis
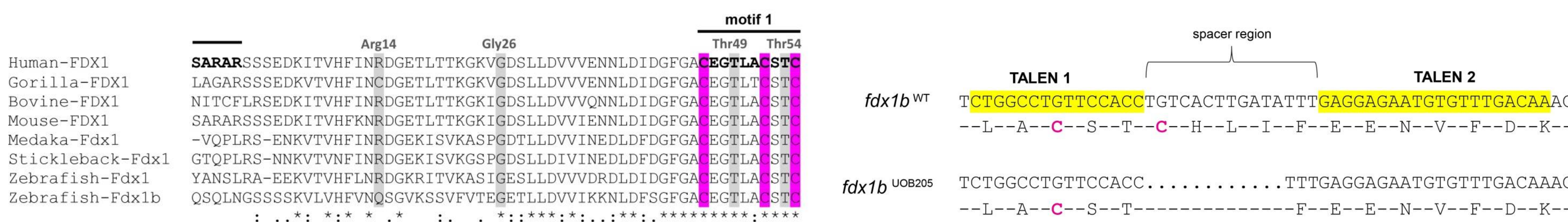

Fdx1b binding TALEN sites target the conserved motif 1 including cysteine residues for $\mathrm{Fe} / \mathrm{S}$ binding
Generation of an allele ( $\left.\mathrm{fd} \times 1 \mathrm{~b}^{\mathrm{UOB} 205}\right)$ with a $12 \mathrm{bp}$ in frame deletion removing a conserved cysteine in motif 1 